\title{
Rule-based Opinion Target and Aspect Extraction to Acquire Affective Knowledge
}

\author{
Stefan Gindl \\ MODUL University Vienna \\ Department of New Media \\ Technology \\ Am Kahlenberg 1 \\ 1190 Vienna, Austria \\ stefan.gindl@modul.ac.at
}

\author{
Albert Weichselbraun \\ University of Applied Sciences \\ Chur \\ Faculty of Information Science \\ Pulvermühlestrasse 67 \\ 7004 Chur \\ albert.weichsel- \\ braun@htwchur.ch
}

\author{
Arno Scharl \\ MODUL University Vienna \\ Department of New Media \\ Technology \\ Am Kahlenberg 1 \\ Vienna Austria \\ arno.scharl@modul.ac.at
}

\begin{abstract}
Opinion holder and opinion target extraction are among the most popular and challenging problems tackled by opinion mining researchers, recognizing the significant business value of such components and their importance for applications such as media monitoring and Web intelligence. This paper describes an approach that combines opinion target extraction with aspect extraction using syntactic patterns. It expands previous work limited by sentence boundaries and includes a heuristic for anaphora resolution to identify targets across sentences. Furthermore, it demonstrates the application of concepts known from research on open information extraction to the identification of relevant opinion aspects. Qualitative analyses performed on a corpus of 100000 Amazon product reviews show that the approach is promising. The extracted opinion targets and aspects are useful for enriching common knowledge resources and opinion mining ontologies, and support practitioners and researchers to identify opinions in document collections.
\end{abstract}

\section{Categories and Subject Descriptors}

H.3.1 [Information Storage and Retrieval]: Content Analysis and Indexing-Linguistic processing; I.2.7 [Artificial Intelligence]: Natural Language Processing-Language parsing and understanding, text analysis

\section{Keywords}

Opinion mining, opinion target extraction, opinion aspect extraction

\section{INTRODUCTION}

Opinion mining has received ample attention over the last decade, representing a core component of a growing number of trend mining, campaign tracking, reputation management and business intelligence tools. The research area covers several different methods, e.g. polarity classification for assigning polarity labels to documents, sentences, or phrases; emotion analysis for retrieving a person's feelings; and opinion holder and target extraction. The latter is capable of

Copyright is held by the International World Wide Web Conference Committee (IW3C2). IW3C2 reserves the right to provide a hyperlink to the author's site if the Material is used in electronic media.

WWW 2013 Companion, May 13-17, 2013, Rio de Janeiro, Brazil. ACM 978-1-4503-2038-2/13/05. pinpointing targets in opinionated sentences. Simple opinion target extraction approaches classify text snippets and apply the overall sentiment value of these snippets to particular entities, e.g. nouns from a whitelist. Such low-level approaches are easy to implement, but founder on grammatical subtleties. These limitations call for more sophisticated approaches able to properly handle grammatical structures such as methods that rely a sentence's dependency tree.

Another important line of research is the identification of different opinion aspects. A digital camera (the opinion target) might become desirable thanks to its long battery life and the high quality of its photos (positive opinion aspects) but might suffer from the drawback of a high weight (a negative opinion aspect). The identification of such aspects delivers valuable data for the producer of the company. Instead of being overwhelmed and confused by the abundance of positive and negative statements on the Web, such techniques are capable of revealing the reason for a positive or negative statement. A not too recent example is Apple's antenna problem in the iPhone 4 . While companies often receive plenty of feedback for popular products, either by angry customers calling their service lines or public media response, they might find it considerably harder to figure out the reasons for a failed product launch, especially if they are not so obvious. Our approach arms every opinion mining toolkit with a powerful mechanism to solve this problem.

In this paper we present an approach for opinion target extraction using syntactic rules. The presented algorithm is based on a subset of the grammar rules defined by [15]. In contrast to the original method, which is limited by sentence boundaries, we utilize heuristic anaphora resolution to identify opinion targets across sentences. A sentiment propagation technique resembling the technique presented in [15] transfers sentiment values from coreferents back to their targets, which allows to determine the sentiment expressed towards a target even if it is not located in the same sentence. In addition, we extend the method for the extraction of opinion targets with an approach for the identification of aspects. Using information extraction patterns in sentences that contain opinion targets we extract these potential aspects. We have assembled a corpus consisting of 100000 product reviews, which are fully parsed for evaluating our target and aspect extraction approach.

The rest of this paper is structured as follows: Section 2 provides a brief overview of open information extraction and 
summarizes existing target extraction approaches, followed by Section 3 that presents details of the developed algorithms and their integration in a reliable processing pipeline. Section 4 shows targets and aspects extracted by the approach, and presents a qualitative assessment based on a number of example sentences and aggregated results that outline the most popular opinion targets and aspects. Section 5 concludes the paper with a summary and outlook on planned future work.

\section{RELATED WORK}

The following section summarizes work on open information extraction and subsequently outlines the current state of the art in the extraction of opinion targets.

\subsection{Open Information Extraction}

Traditionally, open information extraction focuses on the identification of arbitrary and yet unknown relations from extensive Web corpora [4] based on syntactic and lexical constraints such as part-of-speech patterns or heuristic rules regarding the extraction of relevant relations. Such systems usually either start with pre-defined patterns or learn them based on heuristics or training data provided by a supervisor.

The scalability of these methods explains their success on tasks such as the extraction of relations from Wikipedia articles [18], the extraction of common knowledge [10], and the creation of a Wikipedia-based semantic network representing Wikipedia pages as concepts and providing labeled, ontologized relations between these concepts [13].

Fader et al. [5] identify the following shortcomings of current information extraction systems: (i) they often provide uninformative extractions omitting critical information and (ii) tend to yield phrases without meaningful interpretation. The sentence "The company made a deal with its main competitor" might only yield the relation company made - deal and sentences such as "Mr Smith's work was central in uncovering the illegal activities of the company's representative" might return the fragment was central uncovering.

Current research focuses on combining and supervising the relation extraction process with background data obtained from more structured data sources. For example, $\mathrm{Wu}$ and Weld [18] extract such reference data by applying heuristic matching to Wikipedia infobox attributes.

\subsection{Opinion Target Extraction}

Opinion target approaches usually invoke strong linguistic pre-processing. One way to tackle the problem of opinion target extraction is automatic semantic role labeling. Such an approach yields acceptable results, but requires the integration of other strategies such as anaphora resolution [16]. [7] extract opinion targets on multiple domains using conditional random fields. Their approach exploits several features, e.g. simple tokens, part-of-speech and dependency parsing. Nakagawa et al. [14] apply a similar approach using conditional random fields and dependency parsing to Japanese and English sentences. In [17], Sayeed et al. connect a-priori sentiment terms with their targets using syntactical relations, derived from suffix-tree data structures. A crowd-sourcing approach helps to overcome the common problem of data sparseness. Qiu et al. define syntactic rules to identify opinion targets [15]. Their approach propagates the value from opinion-bearing words to their targets. After target identification their algorithm connects them with further terms within the sentence, given that the target and the new term have a dependency relation specified in a predefined set of relations. Thus, a freshly identified target can transfer its sentiment value onto other terms. The terms identified in this second step can either be new targets or unknown sentiment terms, ready for inclusion into a sentiment lexicon. The approach presented in this paper uses a subset of the rules compiled in [15]. Since we focus on target extraction rather than the identification of new sentiment terms, double-propagation, i.e. the bidirectional transfer of sentiment values onto targets and back to unknown sentiment terms, is not relevant for the work presented in this paper.

Instead we leverage anaphora resolution for target extraction. [8] report a positive influence of anaphora resolution on opinion mining tasks. [3] state that off-the-shelve solutions for anaphora resolution are still sparse. Thus, they extend the MARS [12] and CogNIAC [1] tools and employ them for opinion mining. The presented work uses an approach similar to the one presented by [9].

\section{METHODOLOGY}

In contrast to traditional open information extraction techniques, the approach presented in this paper focuses on extracting opinion aspects - i.e., the object of the two abstract relations

1. opinion target $\stackrel{\text { good thanks to }}{\longrightarrow}$ sentiment aspect, and

2. opinion target $\stackrel{\text { bad due to }}{\longrightarrow}$ sentiment aspect.

In other words, we aim at identifying aspects giving hints why the opinion towards the target is positive or negative. The approach consists of two major steps:

- A technique for the propagation of a sentiment charge from a sentiment indicator (i.e. a term from a sentiment lexicon) onto a target. To overcome sentenceboundaries the approach uses a simple heuristic for anaphora resolution.

- An information extraction component allows the discovery of multi-term sentiment aspects, i.e. the objects in the abstract relation above. These sentiment aspects provide reasons for the target's polarity.

The following chapters outline the steps required for the sentiment aspect extraction.

\subsection{Preprocessing}

We use the heuristic preprocessing component of the webLyzard framework ${ }^{1}$ for sentence splitting and tokenization. Subsequently, we label sentiment indicators and determine dependencies with the Stanford parser $^{2}$.

\subsection{Cross-sentence sentiment propagation}

The sentiment propagation component uses the sentiment annotations and the dependency tree from the preprocessing

\footnotetext{
${ }^{1}$ www.weblyzard.com

${ }^{2}$ nlp.stanford.edu/software/lex-parser.shtml
} 
Figure 1: Propagation of sentiment charge from a sentiment indicator, in "The phone has a good screen."

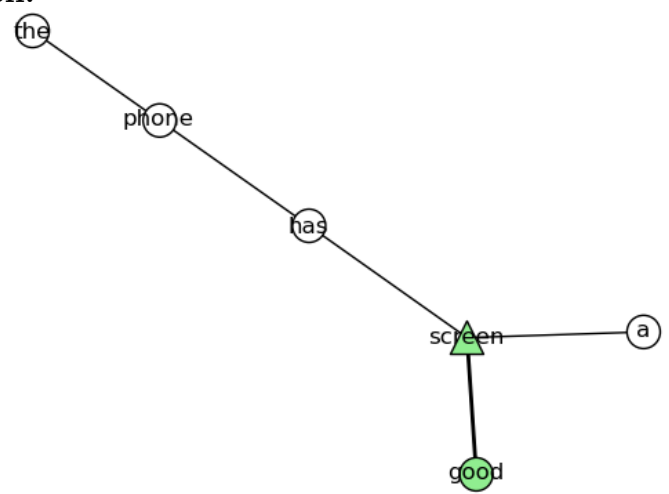

steps and propagates sentiment from the indicators to potential opinion targets. The system uses the first two rules by [15] (single propagation) to identify targets. The first rule propagates a sentiment charge from opinionated terms to noun targets:

$$
O \rightarrow O-D e p \rightarrow T,
$$

s.t. $O \in\{O\}, O-D e p \in\{\mathrm{MR}\}, P O S(\mathrm{~T}) \in\{\mathrm{N}, \mathrm{NN}, \mathrm{NNP}\}$. The application of this rule to the sentence "The phone has a good screen" identifies "good" as an opinionated term and propagates its sentiment charge onto "screen". In other words, "screen" is the target of the "good", as shown in Figure 1 .

Figure 2: Propagation of sentiment charge from a target, in "The iPod is the best mp3 player."

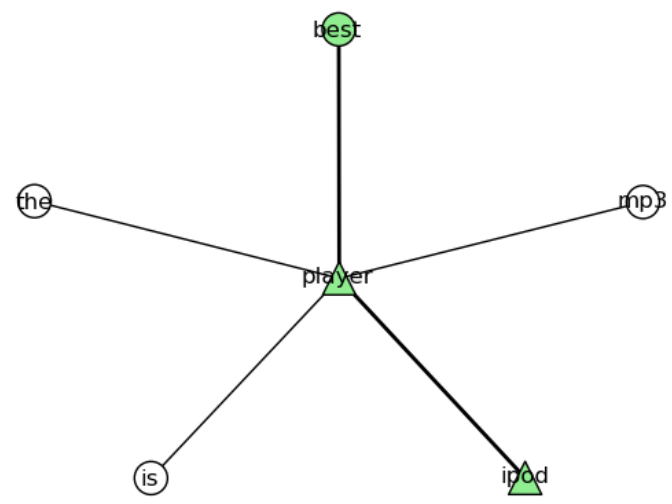

The second rule propagates the sentiment charge of the first identified target onto noun targets in the same sentence:

$$
O \rightarrow O-D e p \rightarrow H \leftarrow T-D e p \leftarrow T,
$$

s.t. $O \in\{O\}, O / T-D e p \in\{\mathrm{MR}\}, \operatorname{POS}(T) \in\{\mathrm{N}\}, \mathrm{NN}$, $\mathrm{NNP}\} ; M R=\{$ advmod, amod, rcmod, nsubj, s, obj, obj2, desc, nn $\}$.

With this additional rule, the system is also able to iteratively identify "player" and "ipod" as the targets in the sentence "The iPod is the best mp3 player.", as demonstrated in Figure 2.
Figure 3: Multiple targets with different charge, in "The phone has a good screen but a bad battery."

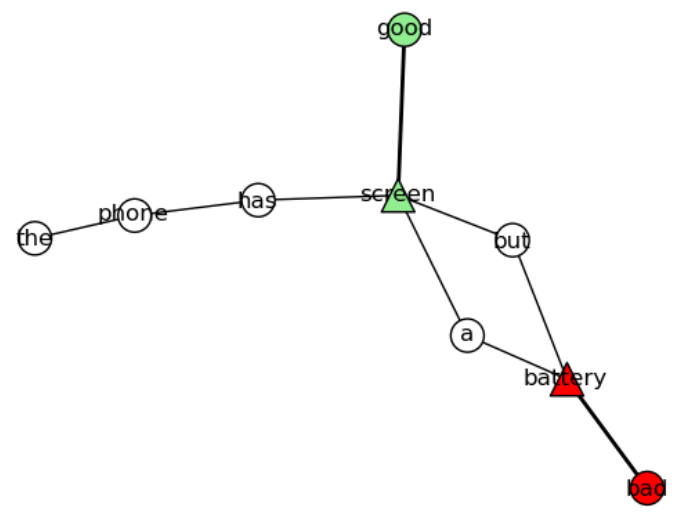

In contrast to simple opinion target extraction approaches that rely on aggregated sentiment values, this sentiment propagation method is able to differentiate between positive and negative sentiment targets within a sentence.

For instance, the sentence "The phone has a good screen but a bad battery" contains two targets, "screen" (with a positive charge) and "battery" (with a negative charge). The aggregated sentiment value for this sentence is neutral, since "good" and "bad" neutralize each other. Approaches that aggregate the sentiment value over the sentences would, therefore, yield a neutral sentiment for both targets. The rules used in the presented approach provide a solution for this problem. They can easily identify "screen" as the target of "good" and "battery" as the target of bad. Figure 3 shows the graph that corresponds to this example.

The two targets of the previous example, "screen" and "battery", can also be considered as opinion aspects. In this paper we distinguish aspects from targets by the presence of a sentiment indicator in the phrase. We only extract subtle aspect, without an a-priori sentiment charge (e.g. "low weight", "long battery life").

The rules presented above identify several targets within one and the same sentence, but are not able to detect targets across sentences. In the two sentences "Yesterday I bought a new phone. It is the best purchase I have ever made." the noun "phone" is clearly a target with a positive charge, although the first sentence does not contain a sentiment indicator. The sentiment charge comes from the second sentence. The mere application of the two rules (as described in 3.2) cannot identify such targets. To overcome this problem we use a heuristic anaphora resolution that has been inspired by prior work of Lau et al. [9]: in case a sentence starts with a personal pronoun, we assume that this pronoun is connected to the last noun in the previous sentence. For the experiments in this paper we limit personal pronouns to the term "it", since it is particularly relevant in the context of our test corpus. If the pronoun has a sentiment charge (propagated by the rules), we propagate its charge back to the noun of the previous sentence.

The heuristic is capable of identifying coreferents across several sentences. Pronouns at the start of a sentence are connected and receive the sentiment charge from the referring sentence. This strategy is also able to detect ambiguous targets. For instance, an author might write about a target 
Table 1: Opinion target extraction patterns for part-of-speech tags. The patterns have been specified using regular expressions.

\begin{tabular}{lll}
\hline Opinion Target Pattern & Part-of-Speech Pattern & Description \\
\hline ADJECTIVE* NOUN+ & $(\mathrm{JJ}(\mathrm{R} \mid \mathrm{S})$ ?) $*(\mathrm{NN}(\mathrm{S}|\mathrm{PS}| \mathrm{P})$ ?)+ & adjectival noun phrase \\
ADVERB ADJECTIVE+ NOUN+ & $(\mathrm{RB}(\mathrm{R} \mid \mathrm{S})$ ?) $(\mathrm{JJ}(\mathrm{R} \mid \mathrm{S})$ ?) $+(\mathrm{NN}(\mathrm{S}|\mathrm{PS}| \mathrm{P})$ ?) + & adverbial noun phrases \\
ADJECTIVE* NOUN PREPOSITION $\backslash$ & $(\mathrm{JJ}(\mathrm{R} \mid \mathrm{S})$ ?) $*(\mathrm{NN}(\mathrm{S}|\mathrm{PS}| \mathrm{P}) ? \mathrm{IN}) \backslash$ & extended noun phrase \\
ADJECTIVE* NOUN+ & $(\mathrm{JJ}(\mathrm{R} \mid \mathrm{S}) ?) *(\mathrm{NN}(\mathrm{S}|\mathrm{PS}| \mathrm{P}) ?)+$ & \\
\hline
\end{tabular}

Figure 4: Cross sentence propagation in "Yesterday $I$ bought a new phone. It is the best purchase $I$ have ever made."

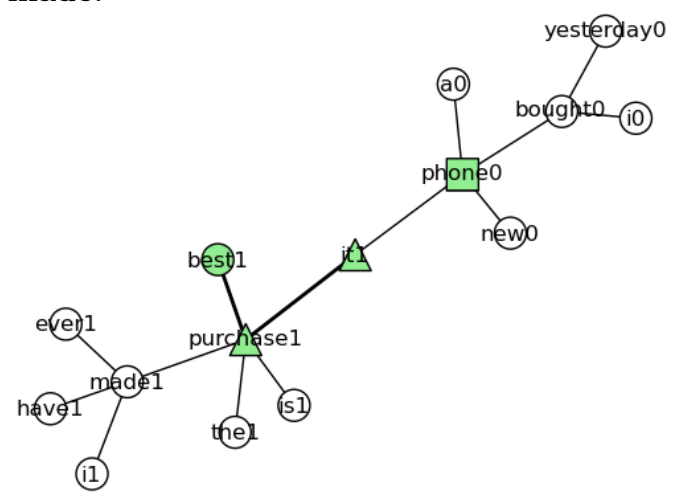

positively at the beginning of a review and might focus on negative aspects towards the end of the document. Figure 5 illustrates this behaviour. The target "phone" has a positive sentiment repeatedly expressed towards it. However, at the end of the text the author also describes negative aspects of the target, rendering its overall sentiment charge ambiguous.

\subsection{Information Extraction Patterns for Sen- timent Aspect Extraction}

One of the main characteristics of open information extraction approaches is their time complexity of $\leq \mathrm{O}(\mathrm{n})$ along with a high precision and a rather low recall (due to the use of extraction rules).

Our research applies the philosophy of open information extraction to opinion target extraction. We have identified the following initial part-of-speech patterns for extracting the objects of implicit sentiment aspect relations. (Table 1). The corresponding part-of-speech patterns listed in the table refer to the Penn Treebank II tag sets [11].

We then use the given patterns for combining multiple part-of-speech tags to opinion aspects. For instance, the phrase ADVERB ADJECTIVE+ NOUN+ yields opinion aspect instances such as highly efficient battery or barely usable software.

In its current stage, our research focuses on the identification of targets and aspects only, but we plan to extend the information extraction component with an iterative pattern learning algorithm that allows us to discover additional extraction patterns based on the identified sentiment targets.

\section{EVALUATION}

This chapter provides a qualitative assessment of the opinion target and aspect extraction method. Our experiments draw upon a corpus of of 100000 Amazon reviews that focus on products from the electronics domain. We consider a star rating below three stars as negative and above three stars as positive. The ratings are distributed equally, i.e. there are 25000 reviews for each star rating except the neutral three stars ratings. We excluded neutral ratings because we expect a more explicit expression of targets and aspects in reviews where the star rating indicates a higher emotive nature.

\subsection{Extraction of Opinion Targets}

The main reason for applying opinion target extraction is its ability to (i) pinpoint opinion targets and (ii) to summarize the targets in a document collection. We processed the review corpus and applied stopword filtering to its output. This resulted in a list of targets an their frequency in the corpus. Table 2 shows the top 20 targets extracted from the corpus and their corresponding frequencies. Without any further post-processing except for stoplist filtering the terms are meaningful given the domain of the corpus, i.e. electronics reviews from Amazon.

Table 2: The top 20 most frequent positive and negative targets with their respective frequency counts.

\begin{tabular}{ll}
\hline Positive targets & Negative targets \\
\hline quality, 7534 & quality, 2708 \\
product, 6429 & product, 2227 \\
price, 4486 & drive, 2043 \\
sound, 4027 & one, 1548 \\
case, 3851 & thing, 1505 \\
one, 2350 & battery, 1315 \\
thing, 2302 & case, 1219 \\
camera, 2258 & sound, 1115 \\
picture, 1823 & design, 1085 \\
screen, 1805 & time, 1072 \\
value, 1624 & screen, 1060 \\
cable, 1549 & cable, 929 \\
battery, 1547 & camera, 906 \\
feature, 1388 & unit, 905 \\
device, 1330 & software, 715 \\
deal, 1328 & price, 712 \\
cover, 1296 & something, 689 \\
way, 1293 & noise, 642 \\
unit, 1269 & plastic, 635 \\
customer, 1230 & way, 607 \\
\hline
\end{tabular}

Looking at example sentences revealed both the strengths as well as weaknesses of the presented approach. Table 3 contains a list of sentences where the anaphora heuristic fired. The first three lines contain working examples, whereas the last two lines contain examples showing the 
Figure 5: Sentiment propagation across several sentences, exposing the ambiguous polarity of the target "phone"; in "My phone is very good. It is light and has a long battery life. It looks sleek and has a crisp and bright screen. However, it is expensive and the sound quality is bad."

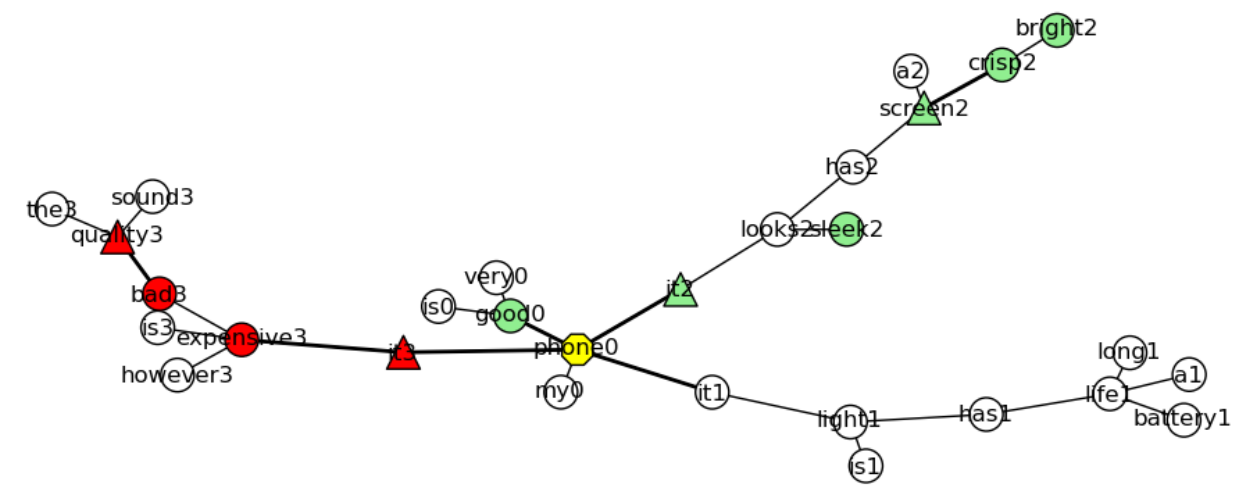

limitation of the heuristic. The first line is an example by an author complaining about a gamepad. While the first sentence contains the sentiment charge in a very subtle way, indiscoverable for the algorithm, the second sentence is more explicit and allows back-propagation using the anaphora heuristic. The second example has a similar structure: the first sentence reveals its sentiment only in a very subtle way whereas the second sentence is far more explicit and thus well-suited for target extraction and sentiment propagation using anaphora resolution.

The third example is worth noting because it shows how the heuristic is able to compensate for the failure of another part of the algorithm. Negation detection is not implemented in that approach, leaving "recommend" untouched and thus ready for propagating a positive value to to "product". However, the system fails to connect these two because of a missing entry in rule dependency set, leaving it neutral where a negative value would have been appropriate. The anaphora heuristic patches this mistake, by backpropagating the negative charge of "waste" onto "product" via "it".

The failed examples in the last two sentences of Table 3 originate from the function of "it" as a dummy pronoun and a placeholder for a noun. Instead of referring to a noun in the previous sentence the function of the pronoun here is merely to provide a noun. The approach cannot detect dummy nouns at the moment but would strongly benefit from the implementation of such a signaling algorithm.

\subsection{Extraction of Opinion Aspects}

The extraction of opinion aspects focuses on phrases in sentences with opinion targets which do not contain any sentiment indicators themselves. Phrases such as "good battery" or "bad lens" are undeniably aspects as well. However, they can be extracted with the extraction methods presented in Section 3.2. The aspect extraction only focuses on "subtle" phrases, i.e. phrases where no sentiment term indicates its polarity. Table 4 shows sentence examples where the extraction algorithm successfully identified aspect. In the first sentence, the positive target "webcam" has the positive aspect of being able to take "crisp photos". The second line is an example for a negative target: the describing aspect "wimpy feather-weight" serves as an indicator for a low-quality power supply. Aspects are intuitively understandable by humans because of their usage of common sense, but they are hard to assess by computers. Since creating a sentiment lexicon containing all potential aspects for numerous task is far outside the scope of any manual approach, the development of scalable automated methods is highly important to create such language resources.

Table 5 contains a list of the 20 strongest positive and negative aspects. We determine "strength" as the ratio of positive and negative occurrences. Here, the approach reveals its weakness. Whereas the examples for positive aspects are meaningful, the examples for negative aspects seem to be senseless or at least very generic.

Table 5: Top 20 strongest positive and negative aspects

\begin{tabular}{ll}
\hline Positive & Negative \\
\hline sound quality & first time \\
light weight & first one \\
sound quality & new one \\
high quality & other reviews \\
digital camera & few days \\
low price & second one \\
little camera & whole thing \\
small size & only problem \\
long battery life & few weeks \\
remote control & many times \\
build quality & few months \\
little device & second time \\
wide angle lens & big deal \\
extra money & only reason \\
audio quality & same thing \\
spare battery & few minutes \\
USB port & other reviewers \\
plus side & few seconds \\
sound reproduction & other users \\
video quality & few hours \\
\hline
\end{tabular}

The current component does not consider negated statements which affects negative sentences, since negative sentiment is often expressed through negation. Another potential reason for the much lower quality of the extracted negative aspects is the strong imbalance between positive and nega- 
Table 3: Examples for successful and failed anaphora resolution.

\begin{tabular}{|c|c|c|}
\hline \multicolumn{3}{|c|}{ Successful anaphora propagation } \\
\hline \multicolumn{3}{|c|}{$\begin{array}{l}\text { I'm playing a lot of Madden NFL } 2004 \text { and after every snap my QB moves now to the left even without touching the pad. } \\
\text { It sucks and makes this product useless. }\end{array}$} \\
\hline \multicolumn{3}{|c|}{ When I bought this Apple charger I thought it was a genuine product. However, it was fake and a bad one a that. } \\
\hline \multicolumn{3}{|c|}{ Bottom line, I wouldn't recommend this product. It was a waste of money and with only a 30 day warranty, I'm stuck. } \\
\hline \multicolumn{3}{|c|}{ Failed anaphora propagation } \\
\hline \multicolumn{3}{|c|}{$\begin{array}{l}\text { While this is supposed to be a class } 10 \text { card, it's so slow in my digital camera that after taking a pic, it takes an extra sec } \\
\text { or two before the camera is readied again. It's very surprising, because an older (class unknown) card that I bought } 3 \\
\text { years ago didn't have the extra sec or two problem in the same digital camera. }\end{array}$} \\
\hline \multicolumn{3}{|c|}{$\begin{array}{l}\text { I have examined every possible aspect for why it is taking so long for renting/downloading movies. It is absolutely } \\
\text { ridiculous...I mean, who gets up in the morning and says "I need to go on Apple TV to rent that movie we want to watch } \\
\text { tomorrow night." }\end{array}$} \\
\hline \multicolumn{3}{|c|}{ Table 4: Example sentences with targets and aspects } \\
\hline Target & Aspect & Sentence \\
\hline webcam $(+)$ & crisp photos & i love the webcam work really well, clear crips photos. \\
\hline power supply (-) & wimpy feather-weight & $\begin{array}{l}\text { Speaking of power, the Sabrent enclosure comes with a wimpy feather- } \\
\text { weight } 12 \mathrm{~V} \text { power supply rated at } 2 \mathrm{~A} \text { bit I really doubt that is is capable } \\
\text { of half that } \ldots\end{array}$ \\
\hline $\operatorname{box}(+)$ & effective heat sink & The metal box itself is already a very effective heat sink for the drive. \\
\hline
\end{tabular}

tive opinion targets. For example, Table 2 shows that the method identified significantly more positive than negative targets. Further experiments will help clarifying this issue and implementing strategies to overcoming this problem (see Section 5). Potential solutions include the refinement of the statistical methods or the inclusion of external resources, such as SenticNet[2], ConceptNet[6], or Freebase.

\subsection{Discussion}

The qualitative analyses performed in this chapter suggest that the targets and aspects extracted by our approach are intuitive and useful. Future experiments and the inclusion of external knowledge will help to remove existing flaws.

Figure 6 shows a visualization of the relations between opinion targets and aspects that have been extracted from the 100000 review corpus using the presented method. The extracted relations are well suited for extending language resources with background information on targets and relevant aspects, and for enriching domain-specific ontologies that may be used to support sentiment analysis and target extraction processes.

In addition, the target and aspect extraction provides relations that can be instrumental in summarizing and visualizing the opinions prevailing in documents or document collections.

\section{CONCLUSION AND FUTURE WORK}

The presented approach uses linguistic rules and anaphora resolution to identify opinion targets in a corpus based on aggregated reviews of electronics products. A subsequent application of part-of-speech information extraction patterns to sentences containing targets reveals aspects of these targets. With this approach we aim at the extraction of subtle aspect without a-priori sentiment charge, such as "long battery life" or "crisp photos".
Figure 6: The target and aspect network extracted from the $\mathbf{1 0 0} 000$ review corpus.

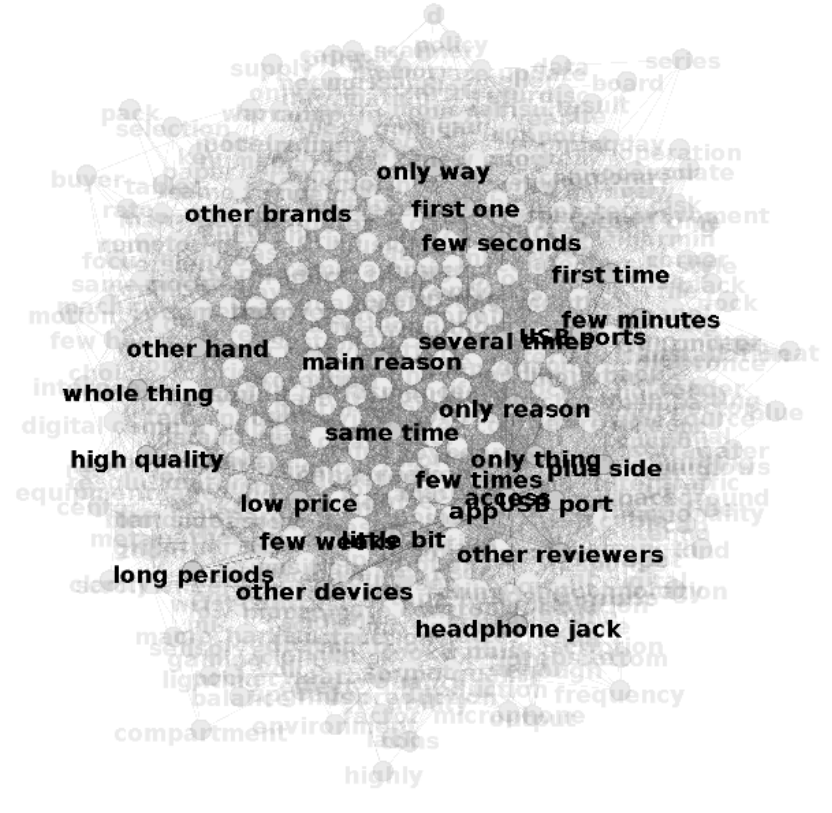


The introduced method yields a network of opinion targets and the corresponding aspects. It facilitates the creation and extension of language resources, and enriches domainspecific ontologies with background information relevant for opinion mining. The extended knowledge resources will contain targets, e.g. products as well as aspects. The aspects, if found in an unknown document, will help to decide if the target obtains a positive or negative sentiment charge. The approach also allows to summarize documents and concisely outline problematic aspects of product. Such a tool is a valuable technique in every toolkit for opinion mining. Future work will focus on optimizing the presented approach by implementing support for the detection of negated statements and providing algorithms for learning aspect extraction patterns based on identified sentiment aspects. From an applied perspective, we plan to use the algorithm across domains and languages. Processing a variety of corpora e.g., news and social media coverage on political events or tourism destinations - in multiple languages will underscore the generic nature of the approach, and help to evaluate and optimize the underlying methods.

\section{Acknowledgement}

The research presented in this paper has been conducted as part of the DIVINE Project (www.weblyzard.com/divine) funded by the Austrian Research Promotion Agency, the WISDOM Project (www.semanticlab.net/wisdom) funded by the Swiss Commission for Technology and Innovation, and the uComp Project (www.ucomp.eu) funded by the Austrian Science Fund within the European CHIST-ERA program line.

\section{REFERENCES}

[1] B. Baldwin. CogNIAC : High Precision Coreference with Limited Knowledge and Linguistic Resources Abstract. In Proceedings of a Workshop on Operational Factors in Practical, Robust Anaphora Resolution for Unrestricted Texts, pages 38-45, Madrid, Spain, 1997.

[2] E. Cambria, R. Speer, C. Havasi, and A. Hussain. SenticNet: A Publicly Available Semantic Resource for Opinion Mining. In AAAI Symposium on Commonsense Knowledge, pages 14-18, 2010.

[3] E. Charniak and M. Elsner. EM Works for Pronoun Anaphora Resolution. In Proceedings of the 12th Conference of the European Chapter of the Association for Computational Linguistics (EACL 2009), pages 148-156, 2009.

[4] O. Etzioni, M. Banko, S. Soderland, and D. S. Weld. Open information extraction from the web. Communications of the ACM, 51(12):68-74, 2008.

[5] A. Fader, S. Soderland, and O. Etzioni. Identifying relations for open information extraction. In Conference on Empirical Methods in Natural Language Processing, 2011.

[6] C. Havasi, S. Street, R. Speer, and J. B. Alonso. ConceptNet 3 : a Flexible, Multilingual Semantic Network for Common Sense Knowledge. In Proceedings of Recent Advances in Natural Language Processing, 2007.

[7] N. Jakob and I. Gurevych. Extracting Opinion Targets in a Single- and Cross-Domain Setting with
Conditional Random Fields. In Proceedings of the Conference on Empirical Methods in Natural Language Processing (EMNLP 2010), pages 1035-1045, 2010.

[8] N. Jakob and I. Gurevych. Using Anaphora Resolution to Improve Opinion Target Identification in Movie Reviews. In Proceedings of the ACL 2010 Conference Short Papers, pages 263-268, Uppsala, Sweden, 2010.

[9] R. Y. Lau, S. S. Y. Liao, K. F. Wong, and D. K. W. Chiu. W 2.0 Environmental Scanning and Adaptive Decision Support for Business Mergers and Acquisitions. MIS Quarterly, 36(4):1239-1268, 2012.

[10] T. Lin, Mausam, and O. Etzioni. Identifying functional relations in web text. In Proceedings of the 2010 Conference on Empirical Methods in Natural Language Processing, EMNLP '10, pages 1266-1276, Stroudsburg, PA, USA, 2010. Association for Computational Linguistics.

[11] M. P. Marcus, M. A. Marcinkiewicz, and B. Santorini. Building a large annotated corpus of english: the penn treebank. Computational Linguistics, 19(2):313-330, June 1993.

[12] R. Mitkov. Robust pronoun resolution with limited knowledge. In Proceedings of the 36th Annual Meeting of the Association for Computational Linguistics and 17th International Conference on Computational Linguistics, pages 869-875, 1998.

[13] A. Moro and R. Navigli. WiSeNet: building a wikipedia-based semantic network with ontologized relations. In Proceedings of the 21st ACM international conference on Information and knowledge management, CIKM '12, pages 1672-1676, New York, NY, USA, 2012. ACM.

[14] T. Nakagawa, K. Inui, and S. Kurohashi. Dependency Tree-based Sentiment Classification using CRFs with Hidden Variables. In Human Language Technologies: The 2010 Annual Conference of the North American Chapter of the Association for Computational Linguistics (HLT), pages 786-794, 2010.

[15] G. Qiu, B. Liu, J. Bu, and C. Chen. Opinion Word Expansion and Target Extraction through Double Propagation. Computational Linguistics, 37(1):9-27, 2011.

[16] J. Ruppenhofer, S. Somasundaran, and J. Wiebe. Finding the Sources and Targets of Subjective Expressions. In Proceedings of the Sixth International Language Resources and Evaluation (LREC), 2008.

[17] A. B. Sayeed, J. Boyd-Graber, B. Rusk, and A. Weinberg. Grammatical Structures for Word-Level Sentiment Detection. In Proceedings of the 2012 Conference of the North American Chapter of the Association for Computational Linguistics: Human Language Technologies (NAACL HLT), pages 667-676, 2012.

[18] Y. Wu, M. Wang, and P. Jin. Disambiguating Dynamic Sentiment Ambiguous Adjectives. In Proceedings of the 23rd International Conference on Computational Linguistics (COLING), pages 1191-1199, Beijing, Oct. 2010. 